\title{
No Further Incidence of Sepsis after Splenectomy for Severe Trauma: A Multi-institutional Experience of The Trauma Registry of THe DGU with 1,630 Patients
}

\author{
M. Heuer ${ }^{1}$, G. Taeger ${ }^{2}$, G. M. Kaiser ${ }^{1}$, D. Nast-Kolb², C. A. Kühne ${ }^{3}$, S. Ruchholtz ${ }^{3}$, R. Lefering ${ }^{4}$, A. Paul ${ }^{1}$, \\ S. Lendemans ${ }^{2}$ and The Trauma Registry of the $\mathrm{DGU}^{5}$ \\ ${ }^{1}$ Department of General-, Visceral- and Transplantation Surgery, ${ }^{2}$ Department of Trauma Surgery, University Hospital Essen, Essen, Germany, \\ ${ }^{3}$ Department of Trauma Surgery, University Hospital Marburg, Marburg, Germany, \\ ${ }^{4}$ Institute for Research in Operative Medicine, Faculty of Medicine, University Witten/Herdecke, Campus Cologne-Merheim, Cologne, Germany, \\ ${ }^{5}$ German Society for Trauma Surgery (DGU), Committee on Emergency Medicine and Intensive Care
}

\begin{abstract}
Objective: Non-operative management of blunt splenic injury in adults has been applied increasingly at the end of the last century. Therefore, the lifelong risk of overwhelming post-splenectomy infection has been the major impetus for preservation of the spleen. However, the prevalence of posttraumatic infection after splenectomy in contrast to a conservative management is still unknown. Objective was to determine if splenectomy is an independent risk factor for the development of posttraumatic sepsis and multi-organ failure.

Methods: 13,433 patients from 113 hospitals were prospective collected from 1993 to 2005 . Patients with an injury severity score $>16$, no isolated head injury, primary admission to a trauma center and splenic injury were included. Data were allocated according to the operative management into 2 groups (splenectomy (I) and conservative managed patients (II)).

Results: From 1,630 patients with splenic injury 758 patients undergoing splenectomy compared with 872 non-splenectomized patients. $96(18.3 \%)$ of the patients with splenectomy and $102(18.5 \%)$ without splenectomy had apparent infection after operation. Additionally, there was no difference in mortality $(24.8 \%$ versus $22.2 \%)$ in both groups. After massive transfusion of red blood cells $(>10)$ non-splenectomy patients showed a significant increase of multi-organ failure (46\% vs. $40 \%$ ) and sepsis (38\% vs. $25 \%$ ).

Conclusions: Non-operative management leads to lower systemic infection rates and mortality in adult patients with moderate blunt splenic injury (grade 1-3) and should therefore be advocated. Patients with grade 4 and 5 injury, patients with massive transfusion of red blood cells and unstable patients should be managed operatively.
\end{abstract}

Key words: blunt abdominal trauma, splenic injury, splenectomy, transfusion, sepsis, mortality

\section{INTRODUCTION}

The incidence of abdominal trauma in patients with multiple injuries is approximately $20 \%$ in Europe, with blunt injuries accounting for $95 \%$ of cases. The solid organs are most frequently affected, with spleen lesions playing a prominent role in the diagnostic and therapeutic management of the blunt abdominal trauma, as they account for approximately $50 \%$ of all injuries to abdominal organs [1, 13, 27, 31].

During the last 15 years, non-operative management of spleen injuries has been clearly demonstrated to be an effective therapeutic option. Six factors predict the failure of non-operative management: hemodynamic instability, preexisting splenic disease, age older than 55 years, grade of injury, size of the hemoperitoneum and contrast blush on CT (computer tomography) scans [7, $11,17]$. Pachter et al commented in his work that patients with grade 4 and 5 injuries were successfully managed non-operatively. The concept of a spleensparing therapy for traumatic injuries has gained importance over recent years, because of rare, but nonetheless possible severe septic postoperative complications. According to several studies, the risk of developing an infection is correlated with the reason for splenectomy and the age of the patient, with the majority of infections occurring years after splenectomy $[4,14,19,28]$. The lifelong risk of overwhelming postsplenectomy infection (OPSI) has been one of the major impetus for preservation of the spleen. However, the prevalence of posttraumatic infection after splenectomy in contrast to a conservative management is still not really known. Some reports and studies refer of higher infection rates after splenectomy in children and adults $[10,16,18]$. But up to now there are no analyses in large patient collectives which altercate with posttraumatic patients against the trauma grade and transfusion of PRBC (Packed Red Blood Cells).

Nonetheless, other authors demonstrated a significantly increase in the risk of failure with splenic injuries [24, 25]. Bleeding, the most common cause of failure can occur at any time of hospitalization. The question remains, whether the non-operative management is always a benefit of the patient? Particularly in consideration of the fact, that potential postoperative risk on the Intensive Care Unit (ICU) such as a major bleeding is disproportionate to the consequences of splenectomy. 
Currently, little is known what effect splenectomy for trauma has on early postoperative infectious complications. It was the aim of the present study to evaluate the infection and MOF rate among 758 patients following splenectomy for multiple traumas compared to 872 patients with non-operative management, based on prospective collected data from The Trauma Registry of the DGU (TR-DGU).

\section{Patients And Methods}

From 1993 until 2005, 13,433 patients from 113 hospitals were documented prospectively in TR-DGU. It is a prospective, standardized and anonymized documentation of severely injured patients at defined time points. This documentation of the clinical course includes the first examination at the accident site (time point A), hospital admission (time point B), transfer to ICU (time point C), and discharge from hospital (time point $\mathrm{D}$ ), together with possible complications (sepsis, organ failure, death) and anatomical and physiological parameters [21]. In this analysis the following eligibility criteria were used:

1. Injury Severity Score (ISS) $\geq 16$

2. Direct admission to a trauma center

3. Splenic injury

Injury Severity Score (ISS) and the severity of individual injuries were determined with the 1998 revision of the Abbreviated Injury Scale (AIS). The AIS is a globally accepted classification system. The classification is based on body regions and each injury is represented by a 7 digit code. The last digit of the code characterizes the injury severity, using a scale ranging from 1 to 6 . AIS 1 represent a minor injury and AIS 6 an injury untreatable according to the latest scientific knowledge, leading mostly to a direct fatal outcome.
Splenic injuries are graded according to the injury scale of the American Association for the Surgery of Trauma (AAST), Table 1. In the present study, the AIS spleen of the Association for the Advancement of Automotive Medicine (AAAM) was chosen as the scale to describe the severity of splenic injury.

Sepsis was defined by the criteria of Bone et al. [3]. The definition of organ failure followed the SOFA score (Sequential Organ Failure Assessment); where an individual organ failure was defined by at least 3 SOFA score points; a multi-organ failure (MOF) was defined as simultaneous failure of at least two organs [29].

All patients with a spleen injury (AIS spleen 2-5) were assigned to the "spleen trauma" group. All remaining patients without spleen injury (AIS spleen 0) were placed in the "non-spleen trauma" group.

The restriction to cases with ISS $\geq 16$ guaranteed a minimum injury severity of AIS 3 for the primary region in the respective study groups.

In order to assess the risk of death based on the initial severity of injury, a prognosis was made using the Revised Injury Severity Classification (RISC). Until 2003, TR-DGU has been using the TRISS Score, an internationally-spread score system for the prognosis of trauma patients based on the American MTOS study. Some studies on prognostically relevant factors in trauma as well as criticism by other authors concerning TRISS have lead to the development of a new severity classification system for the prognosis of outcome, using the data of TR-DGU. With data of more than 2000 patients and multivariate statistical modeling, the RISC has been developed and repeatedly validated within the register. The RISC takes into consideration: the age, the anatomical pattern of injuries (New ISS), the head injury, the severe pelvis trauma, coagulation (PT'), the base excess, three indirect signs of bleeding (hypotension, low hemoglobin, mass-

Table 1. AAST-scale and modified scale for classification of spleen injuries.

\begin{tabular}{|c|c|c|c|}
\hline AAST Grade & Injury & Injury Description & AIS-98* Grade \\
\hline \multirow{2}{*}{$\mathrm{I}$} & hematoma & subcapsular, $<10 \%$ surface & 2 \\
\hline & laceration & capsular tear, $<1 \mathrm{~cm}$ & 2 \\
\hline \multirow{3}{*}{ II } & hematoma & subcapsular, $10-50 \%$ surface; & \\
\hline & & intraparenchymal hematoma, $<10 \mathrm{~cm}$ in diameter & 2 \\
\hline & laceration & $1-3 \mathrm{~cm}$ deep, $<10 \mathrm{~cm}$ long & 2 \\
\hline \multirow{2}{*}{ III } & hematoma & subcapsular, $>50 \%$ surface; intraparenchymal hematoma, $>10 \mathrm{~cm}$ & 3 \\
\hline & laceration & $>3 \mathrm{~cm}$ parenchymal & 3 \\
\hline IV & laceration & parenchymal disruption involving $25-75 \%$ of spleen & 4 \\
\hline \multirow{2}{*}{$\mathrm{V}$} & laceration & parenchymal disruption involving $>75 \%$ of spleen & 5 \\
\hline & vascular & splenic venous injuries & 5 \\
\hline VI & vascular & splenic avulsion & 6 \\
\hline
\end{tabular}

*Note-AIS-98 = Abbreviated Injury Scale, 1998 version. 
transfusion) as well as cardiac arrest. Comparable analysis have shown that the RISC is significantly superior to the scores (e.g. TRISS) used so far. Since 2003, the RISC is being used as main instrument for severity adjustment and outcome analysis [8, 22].

\section{STATISTICS}

From 1993 until 2001, data were collected and entered on paper sheets. Since 2002, data collection was done with internet-based data entry software with integrated plausibility checks. The anonymized data were analyzed with the statistical software SPSS Version 14, Chicago, USA). Incidences are presented with counts and percentages, continuous values with mean and standard deviation (SD). Analysis was mainly restricted to descriptive statistics. Statistical tests were avoided due to the multiple comparisons (several groups and outcome parameters), as well as the high sample size which could lead to irrelevant significances. In selected situations only, data from the group with spleen trauma were compared statistically against the remaining groups $\left(\mathrm{Chi}^{2}\right.$ test for incidence rates and U-test for continuous values).

\section{RESULTS}

From 1993 to 2005, a total of 13,433 emergency room patients with an ISS $\geq 16$ points were included in TR-
DGU, of whom 1,630 (12.1\%) matched the inclusion criteria of the present study. Of these, 295 (18.1\%) had splenic lesions classified as AIS spleen 2, 457 $(28.0 \%)$ as AIS spleen 3, $485(29.8 \%)$ as AIS spleen 4 and $393(24.1 \%)$ as AIS spleen 5 were identified. Laparotomy with splenectomy was required in 758 $(46.5 \%)$ patients with splenic injury. 32 (10.8\%) splenectomies of AIS spleen 2, $106(23.2 \%)$ of AIS spleen 3, $316(65.2 \%)$ AIS spleen 4 and $304(77.4 \%)$ of AIS spleen 5 were performed, Table 2 . Average age in the total sample was 35.38 years and $71.3 \%$ of the included subjects were male. The mean ISS was 38.94 points.

\section{MORTALITY}

Mortality after splenectomy was slightly increased $(24.8 \%)$ compared to patients without splenectomy $(22.2 \%)$, Table 3 . Supporting analysis of these results between the splenectomized and the non-splenectomized patients showed that the comparable mortality is explained almost by the same, without being equal: AIS scores head: $37.7 \%$ vs. $43.9 \%$, thorax: $74.8 \%$ vs. $78.0 \%$ and extremities: $46.2 \%$ vs. $47.1 \%$. Adjusting for severity with the RISC Score shows that patients with splenic injury do not die more frequently than expected. The $25.0 \%$ mortality observed $(95.0 \%$ confidence interval 27.6 - 38.4) in the patients after splenectomy offsets a prognostic mortality rate of

Table 2. Data analyses of splenic injured patients indexed by AIS spleen and followed treatment option, splenectomy or nonsplenectomy.

\begin{tabular}{|c|c|c|c|c|c|c|c|c|}
\hline \multirow[t]{2}{*}{ AIS spleen } & \multicolumn{2}{|c|}{ Patients } & \multicolumn{2}{|c|}{ Splenic Injury } & \multicolumn{2}{|c|}{ Splenectomy } & \multicolumn{2}{|c|}{ Non-Splenectomy } \\
\hline & $\mathrm{n}$ & $\%$ & n & $\%$ & $\mathrm{n}$ & $\% *$ & $\mathbf{n}$ & $\% *$ \\
\hline 0 & 11,803 & 87.9 & 0 & 0 & 0 & 0 & 0 & 0 \\
\hline 2 & 1,630 & 12.1 & 295 & 18.1 & 32 & 10.8 & 263 & 89.2 \\
\hline 3 & & & 457 & 28 & 106 & 23.2 & 351 & 76.8 \\
\hline 4 & & & 485 & 29.8 & 316 & 65.2 & 169 & 34.8 \\
\hline 5 & & & 393 & 24.1 & 304 & 77.4 & 89 & 22.6 \\
\hline total & 13,433 & 100 & 1,630 & 12.1 & 758 & 5.6 & 872 & 94.4 \\
\hline
\end{tabular}

$* \% /$ max AIS spleen

Table 3. General data analyses of studied splenic injured patients indexed by treatment option.

\begin{tabular}{lccc}
\hline & $\begin{array}{c}\text { Unit } \\
\mathrm{n}=758\end{array}$ & $\begin{array}{c}\text { Splenectomy } \\
\mathrm{n}=872\end{array}$ & Non-Splenectomy \\
\hline ISS & points & 41.6 & 36.5 \\
Age & years & 36.5 & 34.4 \\
Male & $\%$ & 71.4 & 71.3 \\
Mortality & $\%$ & 24.8 & 22.2 \\
AIS head $\geq 3$ & $\%$ & 37.7 & 43.9 \\
AIS thorax $\geq 3$ & $\%$ & 74.8 & 78.0 \\
AIS extremities $\geq 3$ & $\%$ & 46.2 & 47.1 \\
ICU & days & 16.9 & 16.3 \\
Hospital stay & days & 31.4 & 28.7
\end{tabular}


26.7\% (SMR 0.94). Patients without splenectomy showed a slightly lower mortality rate compared with patients after splenectomy (Mortality 21.5, RISC 23.0, SMR 0.94). In both groups of injuries, prognosticated mortality did not deviate from the observed mortality, Table 4. Even more, the prognosticated mortality was lower in splenectomized than in non-splenectomized patients. Consequently, there was a lower overall mortality after splenectomy. At the same time, the subanalysis carried out shows that non-splenectomized patients with AIS score 2-3 have lower mortality rates - patients with AIS spleen score 4-5 have significantly worse rates however - than patients after splenectomy, Table 5. The same results are shown considering the sepsis and MOF-rates.

\section{Blood Transfusion and Incidence of Shock}

Splenectomized patients had a higher need for massive blood transfusions (number of transfused PRBC $>10)$, (31.9\% vs. $19.5 \%)$, Table 4. The high blood loss in the splenectomy group is correlated with the blood pressure pattern in the emergency room (ER). Initial blood pressure $\leq 90 \mathrm{mmHg}$ was $31.7 \%$ in of the splenectomy group and $25.9 \%$ of the non-splenecto- my group. In the ER, an initial hemoglobin content of less than $9 \mathrm{mg} / \mathrm{dl}$ was much more frequent in the splenectomy group $(42.6 \%)$ than in the non-splenectomy group $(31.7 \%)$. Analogous to this, the average amount of transfused PRBC until admission to the intensive care unit was much higher in the group of $\mathrm{pa}$ tients with splenectomy ( 8.5 units) compared to the non-splenectomy group (5.0 units).

\section{SEPSis AND Organ FAILURE}

Both groups nearly showed the same mortality after 24 hours $(14.1 \%$ vs. $13.5 \%)$. Furthermore, patients with a splenectomy showed near by the same average late mortality of $24.8 \%$ as those without splenectomy $22.2 \%$. One cause for the comparable mortality in comparison with patients with non splenectomy is possibly the same sepsis rate $(18.3 \%$ vs. $18.4 \%)$, if the first 24 hours were survived, Table 4 . The assimilable sepsis rate in both groups is also reflected in the frequency of $\mathrm{OF}(53.0 \%$ vs. $45.6 \%)$ and $\mathrm{MOF}(33.4 \%$ vs. 29.0).

As presented in Table 5, the above described separation of patients in dependence of the AIS spleen scores is shown. Non-splenectomy patients with an

Table 4. Blood transfusion, sepsis and mortality analyses of studied splenic injured patients.

\begin{tabular}{lccc}
\hline & Unit & $\begin{array}{c}\text { Splenectomy } \\
\mathrm{n}=758\end{array}$ & $\begin{array}{c}\text { Non-Splenectomy } \\
\mathrm{n}=872\end{array}$ \\
\hline PRBC & units & 8.5 & 5.0 \\
$>$ 10 PRBC & $\%$ & 31.9 & 19.5 \\
RR $\leq \mathbf{9 0 m m H g}$ (ER) & $\%$ & 31.7 & 25.9 \\
Hb <9mg/d1 & $\%$ & 42.6 & 31.7 \\
Organ failure & $\%$ & 53.0 & 45.6 \\
Multiple Organ Failure & $\%$ & 33.4 & 29.0 \\
Sepsis & $\%$ & 18.3 & 18.4 \\
Mortality within 24 hours & $\%$ & 14.1 & 13.5 \\
& & $\mathrm{n}=711$ & $\mathrm{n}=805$ \\
Mortality & $\%$ & 25.0 & 21.5 \\
RISC & $\%$ & 26.7 & 23.0 \\
SMR & & 0.94 & 0.94 \\
\hline
\end{tabular}

Table 5. Mortality by AIS spleen and treatment group when survived.

\begin{tabular}{cccccccc}
\hline AIS spleen & Splenectomy & N & ISS & OF (\%) & MOF (\%) & Sepsis (\%) & Mortality (\%) \\
\hline \multirow{2}{*}{2} & Yes & 32 & 32.4 & 76.5 & 71.6 & 26 & 19 \\
& No & 263 & 31.2 & 47.7 & 30.6 & 17 & 12 \\
3 & Yes & 106 & 33.4 & 72.7 & 53.2 & 26 & 23 \\
& No & 351 & 35.1 & 56.6 & 35.9 & 20 & 19 \\
4 & Yes & 316 & 39.5 & 64.4 & 43.0 & 18 & 21 \\
& No & 169 & 39.7 & 67.8 & 41.1 & 20 & 25 \\
& Yes & 304 & 47.9 & 76.0 & 41.0 & 16 & 30 \\
& No & 89 & 52.1 & 96.0 & 72.2 & 88 & 58 \\
\hline
\end{tabular}


Table 6. When survival after 24 hours and more then 10 PRBC.

\begin{tabular}{lcccccc}
\hline & ISS & PRBC & OF (\%) & MOF (\%) & Sepsis (\%) \\
\hline Splenectomy & 42.7 & 18.7 & 64 & 40 & 25 & 125 \\
Non- Splenectomy & 40.4 & 18.6 & 59 & 46 & 38 & 95 \\
\hline
\end{tabular}

AIS spleen 2-3 show lower sepsis as well as OF- and MOF-rates as patients after splenectomy. With a spleen score of 4 and 5 however, this is just the other way around: sepsis-, OF- and MOF-rates are significantly better than after conducted splenectomy.

In order to assess, whether an increased administration of PRBC has an influence on sepsis-, MOFand/or OF-rates, a subgroup was formed consisting of "only" transfused patients with $\geq 10$ units who survived the first 24 hours. The results in this subgroup are displayed in Table 6. With an almost identical administration of PRBC in splenectomy patients ( $\mathrm{n}=$ $125)$ as well as non-splenectomy patients $(\mathrm{n}=95)-$ units 18.7 vs. 18.6 - significant differences in the sepsis- $(25 \%$ vs. $38 \%)$ and MOF-rate were shown. Consequently, only severely injured patients seemed to profit from operative care.

\section{DisCUSSION}

The modern era for splenic surgery for starts 1892 when Riegner reported a splenectomy in a 14-year old worker after blunt trauma. This report set the stage for routine splenectomy, which was performed for all splenic injury in the next generations. Despite early reports by Pearce and by Morris and Bullock that splenectomy in animals caused impaired defenses against infection, little challenge to routine splenectomy was made until King and Schumacker in 1952 reported a syndrome of OPSI [12]. Many studies have since demonstrated the importance of the spleen in preventing infections, particularly from the encapsulated organisms. The most serious of these infections is the syndrome of OPSI, which occurs rarely $(0.5 \%)$ in adults subjected to splenectomy but carries a prohibitive mortality in unvaccinated patients. Therefore, the preservation of the spleen and the shift form conventional operative management to selective non-operative management of blunt splenic trauma injuries has shown a noticeable trend varied from $53 \%$ to $77 \%$ in the past decade with failure rates generally ranging from $2 \%$ to $11 \%[9,15,20]$. Increasing awareness of the risk of OPSI and postoperative complications were the major incentives for the concept of a spleen- sparing therapy for traumatic injuries. The precise incidence of OPSI after splenectomy for trauma remains controversial, and published estimates very considerably. An epidemiologic study of 1490 patients who underwent splenectomy in Western Australia over 12 years examined a trauma cohort $(n=628)$ that was included. After trauma, the incidence and mortality of late OPSI was 0.03 per 100 person year's exposure [6]. However, the incidence of OPSI after splenectomy for trauma is still not known.

Currently, the standard of care for post-splenectomy patients includes immunization with polyvalent pneumococcal vaccine (PPV 23, H. influenza type b conjugate and meningococcal polysaccharide vaccine) within 2 weeks of splenectomy [26]. Despite this established standard the literature reflects a diverse 11 to $75 \%$ post-splenectomy immunization rate [23]. Thus, a standardized immunization behavior would further improve the outcome after splenectomy. Initially, one has come to a different conclusion. A paradigm shift in the 90ies hence led to an improved outcome after splenic trauma. During the observation period of this study (1993-2000) there was a fundamental shift to more conservative managed splenic injuries, Table 7 . The increasing preservation rate from $51.2 \%$ to $39.6 \%$ leads to a lower mortality rate from $27.3 \%$ to $20.3 \%$. Improved survival rates during this time superposed the immunization behavior and finally led to an improvement oft he total outcome [2].

Despite the known risk of OPSI, there are risk factors associated with the non-operative treatment regimen. Cocanour examined the incidence and type of delayed complications from non-operative management of adult splenic injury. Therefore, 280 patients were admitted with blunt splenic injury. The mean age was $32.2 \pm 1.0$ years and the mean ISS was $22.8 \pm 0.9$. Fifty-nine patients $(21 \%)$ died of multiple injuries within 48 hours and were eliminated from the study. $134(48 \%)$ patients were treated operatively within the first 48 hours after injury and 87 (31\%) patients were managed non-operatively. Cocanour et al reviewed the number of PRBC transfused, ICU length of stay, overall length of stay, outcome, and complications occurring more than 48 hours after injury directly attributable to the splenic injury. The study demonstrated

Table 7. Increase of nonoperative management from 1993 to 2005.

\begin{tabular}{lllcc}
\hline Time period & N & ISS & Mortality (\%) & Splenectomy (\%) \\
\hline $1993-2000$ & 535 & 40.3 & 27.3 & 51.2 \\
$2001-2003$ & 641 & 39.1 & 22.5 & 47.4 \\
$2004-2005$ & 453 & 37.0 & 20.3 & 39.6 \\
\hline
\end{tabular}


that patients managed non-operatively had a significantly lower ISS $(p<0.5)$ than patients treated operatively. Length of stay was significantly decreased in both the number of ICU days as well as total length of stay $(p<0.5)$. The number of PRBC transfused was also significantly decreased in patients managed nonoperatively $(p<0.5)$. In conclusion, a significant number of delayed splenic complications occurred during the non-operative management of splenic injuries and were potentially life-threatening [5].

Furthermore, the recognition of infections and other risks associated with blood transfusion, which may be required with non-operative management, has led to a higher threshold to avoid transfusions and, thus, tolerate lower hematocrits. Luna and Dellinger analyzed the risk of death from post-transfusion hepatitis per PRBC transfused to be $0.14 \%$ and death from OPSI at $0.026 \%$ of adults who undergo splenectomy and $0.052 \%$ of children who undergo splenectomy. Based on these figures, the conditional probability of death in a child who initially undergoes non-operative observation therapy is $0.17 \%$, compared with $0.06 \%$ for initial operative therapy. In adults, $0.26 \%$ of observed patients die, compared with $0.06 \%$ for those operated initially.

In our study we were able to demonstrate the standardized mortality rate for the RISC score was $0.93 \%$ in the group with splenectomy $(\mathrm{n}=758)$ and $0.97 \%$ in the group without splenectomy $(n=872)$. Our study showed a survival rate of $22.2 \%$ in the non-operative cohort, which was lower than in the operative group $(24.8 \%)$. Similar conclusions are also found in other studies. Our overall results showed no differences in the rate of postoperative infectious complications in patients without splenectomy $(18.4 \%)$, compared with those who were managed operatively $(18.3 \%)$.

The study presented could show an AIS spleen score-dependent distribution of the various rates in splenectomy and non-splenectomy patients. At that, non-splenectomy patients with AIS spleen score 2-3 had better sepsis-, OF- and MOF-rates as well as mortality rates. Conversely, these patients did not profit from conservative management when the AIS spleen score was 4-5. In that case, splenectomy patients showed significantly better rates over all sub-analyses studied. Reverse results were obtained by Wiseman and colleagues. They reviewed all trauma patients undergoing splenectomy in a two year period. Though, each splenectomy patient was matched to a unique trauma patient who underwent laparotomy without splenectomy based on age, gender, mechanism of injury, ISS, and presence of colon or other hollow visceral injury. There were 98 splenectomy patients and 98 controls. The splenectomy patients had more overall infectious complications ( $45 \%$ vs. $30 \%, \mathrm{p}=0.04$ ) trended toward more urinary tract infections (12\% vs. $5 \%, p=0.12)$, and more often had pneumonia $(30 \%$ vs. $14 \%, \mathrm{p}=0.02)$. Additionally, more splenectomy patients developed multiple infections (20\% vs. $7 \%$, p $=0.01)$. Like us, they found no significant differences in mortality $(11 \%$ vs. $8 \%, \mathrm{p}=0.63)$. Recapitulatory, Wiseman et al postulated that splenectomy is associated with an increase in infectious complications after laparotomy for trauma. This increase in infections is not associated with increased mortality [30]. Also Gauer et al., as mentioned before, describe an increased infection rate after performed splenectomy in their youngest study 2008. In this, although prospective study, only 155 patients could be included. No sub-group analysis in dependence of the respective grade of trauma was carried out. Hence, only a very general statement regarding the total collective could be given, not however describe the essential AIS spleen score-dependent differences in the splenic injured - as presented by us. In addition, higher rates of sepsis, OF and MOF can also always be triggered by increased loss of blood. However, we could not assess increased mortality even in average 8.5 units/patient after performed splenectomy due to trauma in comparison to non-splenectomy patients with significantly lower PRBC-substitution (5 units/patient).

In contrast, we were able to show that despite an increased application of PRBC and at the same time high, however identical ISS in both patient collectives the outcome due to splenectomy will only be improved, Table 6. After synopsis of the results, conservative procedure is gold standard, however with highlevel injuries (>AIS spleen 4) and continuing blood loss, a conservative procedure may not be forced. In this case, splenectomy should be performed without further delay. As a specific risk for the OPSI-syndrome is not known but presumably low, the splenic preservation still has first priority; a respective immunization, however, has to be demanded.

\section{CONCLUSION}

Non-operative management leads to lower systemic infection rates and mortality in adult patients with moderate blunt splenic injury (Grade 2-3) and should therefore be advocated. Patients with Grade 4 and 5 injury, patients with massive transfusion of PRBC and unstable patients should be managed operatively as soon as possible to prevent further development of hemorrhaging shock.

Acknowledgements: The authors thank the German Society of Trauma Surgery, Berlin, Germany, for the permission to analyze data from The Trauma Registry, and acknowledge the large amount of work by parties involved centers who reported patient data to the project.

Participating hospitals: Universitätsklinik der RWTH Aachen, Zentralklinikum Augsburg, Kreiskrankenhaus Bad Hersfeld, SANA Krankenhaus Bergen / Rügen, Charité - Campus Virchow-Klinikum Berlin, Martin-Luther-Krankenhaus Berlin, Vivantes Klinikum Berlin Friedrichshain, Klinikum BerlinBuch, BG-Unfallklinik Berlin-Mahrzahn, Krankenanstalten Gilead Bielefeld, Südeifel-Kliniken Bitburg, BG-Klinik Bochum Bergmannsheil, Knappschaftskrankenhaus der Ruhr-Universität Bochum, Friedrich-Wilhelms-Universität Bonn, Städt. Klinikum Braunschweig, Zentralkrankenhaus Sankt-Jürgen-Straße Bremen, Zentralkrankenhaus Bremen Ost, Klinikum Bremerhaven-Reinkenheide, Landeskrankenhaus Bruck/Mur (Austria), Kreiskrankenhaus Burg, Allgemeines Krankenhaus Celle, Klinikum Chemnitz, Städt. Klinikum Dessau, Klinikum Lippe-Detmold, Detmold, Krankenhaus Dresden-Neustadt, Technische Universität Dresden, Krankenhaus Dresden-Friedrichstadt, Heinrich- 
Heine-Universität Düsseldorf, Krankenhaus der Barmherzigen Brüder Eisenstadt (Austria), Klinikum Erfurt, Kreiskrankenhaus Eschwege, Universitätsklinikum Essen, Evang. Krankenhaus Lutherhaus Essen, BG Unfallklinik Frankfurt / Main, Universitätsklinik Frankfurt / Main, Klinikum Frankfurt/Oder, Herz-Jesu-Krankenhaus Fulda, Klinikum Fürth, Johanniter-Krankenhaus Geesthacht, Städtisches Klinikum Görlitz, Klinik an Eichert Göppingen, Georg-August-Universität Göttingen, Universität Graz (Austria), Allg. Unfallvericherungsanstalt Graz (Austria), Kreiskrankenhaus Grevenbroich, Universitätsklinik Groningen (Netherland), Kreiskrankenhaus Gummersbach, BG Klinik Bergmannstrost Halle/Saale, BG-Unfallkrankenhaus Hamburg, Universitätsklinik Hamburg-Eppendorf, Kreiskrankenhaus Hameln, Medizinische Hochschule Hannover, Krankenhaus Hannover-Nordstadt, Friederikenstift Hannover, Ev. Krankenhaus Hattingen, Orthopäd. Universitätsklinik Heidelberg, St. Bernward Krankenhaus Hildesheim, Universität des Saarlandes Homburg / Saar, Waldviertel Klinikum Horn (Austria), LKH Judenburg-Knittelfeld (Austria), Städt. Klinikum Karlsruhe, Christian-Albrechts-Universität Kiel, Bundeswehrkrankenhaus Koblenz, Unfallchirurgische Klinik der Universität zu Köln, Städt. Klinikum Köln-Merheim, Allg. öff. Krankenhaus Krems/Donau (Austria), Städt. Klinikum St. Georg Leipzig, Universität Leipzig, Ev. Krankenhaus Lengerich, Allg. öffentl. Krankenhaus Linz (Austria), Ev. Krankenhaus Lippstadt, Universitätsklinikum Lübeck, BG Unfallklinik Ludwigshafen, St.-Marien-Hospital Lünen, Krankenhaus Altstadt, Städt. Klinikum Magdeburg, Otto-von-Guericke-Universität Magdeburg, Johannes-Gutenberg-Universität Mainz, Universitätsklinikum Mannheim, Universität Marburg, Heilig Hart Ziekenhuis Roeselare, Menen (Belgium), Klinikum Minden, Krankenhaus Maria Hilf Mönchengladbach, Klinikum Großhadern der LMU München, Klinikum Innenstadt der LMU München, Städt. Krankenhaus München-Bogenhausen, Städt. Krankenhaus München-Harlaching, Westfälische Wilhelms-Universität Münster, BG-Unfallklinik Murnau, Lukaskrankenhaus der Städt. Kliniken Neuss, Marienhospital Osnabrück, Vogtland Klinikum Plauen, Klinikum Remscheid, Klinikum Rosenheim, St. Johanns-Spital Landeskrankenhaus Salzburg (Austria), Diakonissenkrankenhaus Schwäbisch Hall, Kreiskrankenhaus Soltau, Johanniter-Krankenhaus der Altmark Stendal, Klinikum St. Elisabeth Straubing, Kreiskrankenhaus Tirschenreuth, Kreiskrankenhaus Traunstein, BG-Unfallklinik Tübingen, Bundeswehrkrankenhaus Ulm, Universitätsklinik Ulm, Klinikum der Stadt VillingenSchwenningen, Klinikum Weiden/Oberpfalz, Asklepios Kreiskrankenhaus Weißenfels, Donauspital Wien (Austria), Ferdinand-Sauerbruch-Klinikum Wuppertal, Helios Klinikum Wuppertal, Julius-Maximilians-Universität Würzburg, Universitätsspital ETH Zürich (Switzerland), Rettungsstelle Zusmarshausen

\section{REFERENCES}

1. Aufmkolk M, Nast-Kolb D. Abdominaltrauma. Unfallchirurg 2001; 72: 861-875.

2. Bain IM, Kirby RM. 10 year experience of splenic injury: an increasing place for conservative management after blunt trauma. Injury 1998; 29: 177-182.

3. Bone RC, Balk RA, Cerra FB. Definitions for sepsis and organ failure and guidelines for the use of innovative therapies in sepsis. The ACCP/SCCM Consensus Conference Committee. American College of Chest Physicians/Society of Critical Care Medicine. Chest 1992; 101: 1644-1655.

4. Cadeddu M, Garnett A, Al-Anezi K, et al. Management of spleen injuries in the adult trauma population: a ten year experience. Can J Surg 2006; 49: 386-390.
5. Cocanour CS, Moore FA, Waren DN, et al. Delayed complications of nonoperative management of blunt adult splenic trauma. Arch Surg 1998; 133: 619-625.

6. Cullingford GL, Watkins DN, Watts AD, et al. Severe late postsplenectomy infection. Br J Surg 1991; 78: 716721.

7. Davis KA, Fabian TC, Croce MA, et al. Improved success in nonoperative management of blunt splenic injuries: embolization of splenic artery pseudoaneurysms. J Trauma 1998; 44: 1008-1015.

8. Dutton RP, Lefering R, Lynn M. Database predictors of transfusion and mortality. J Trauma 2006; 60: 70-77.

9. Eigenberger K, Sillaber C, Greitbauer M, et al. Antibody responses to pneumococcal and hemophilus vaccinations in splenectomized patients with hematological malignancies or trauma. Wien Klin Wochenschr 2007; 119: 228234.

10. Gauer JM, Gerber-Paulet S, Seiler C, et al. Twenty years of splenic preservation in trauma: lower early infection rate than in splenectomy. World J Surg 2008; 32: 27302735.

11. Harbrecht BG, Zenati MS, Ochoa JB, et al. Evaluation of a 15-year experience with splenic injuries in a state trauma system. Surgery 2007; 141: 229-238.

12. King H, Shumacker HB Jr. Splenic studies: I. susceptibility to infection after splenectomy performed in infancy. Ann Surg 1952; 136: 239-242.

13. Kollig E, Muhr G. Abdominale und thorakale Organverletzungen. Trauma Berufskrankh 2001; 3: 388-395.

14. Lucas CE. Splenic trauma. Choice of management. Ann Surg 1991; 213: 98-112.

15. Miller PR, Croce MA, Bee TK, et al. Associated injuries in blunt solid organ trauma: implications for missed injury in nonoperative management. J Trauma 2002; 53: 238242.

16. O'Neal BJ, McDonald JC. The risk of sepsis in the asplenic adult. Ann Surg 1981; 194: 775-778.

17. Pachter HL, Guth AA, Hofstetter SR, et al. Changing patterns in the management of splenic trauma: the impact of nonoperative management. Ann Surg 1998; 227: 708-719.

18. Pimpl W, Dapunt O, Kaindl H, et al. Incidence of septic and thromboembolic-related deaths after splenectomy in adults. Br J Surg 1989; 76: 517-521.

19. Reihner E, Brismar B. Management of splenic trauma changing concepts. Eur J Emerg Med 1995; 2: 47-51.

20. Robinson WP, Ahn J, Stiffler A, et al. Blood transfusion is an independent predictor of increased mortality in nonoperatively managed blunt hepatic and splenic injuries. J Trauma 2005; 58: 437-445.

21. Ruchholtz S. Das Traumaregister der DGU als Grundlage des interklinischen Qualitätsmanagements in der Schwereverletztenversorgung - Eine Multicenterstudie. Unfallchirurg 2000; 103: 30-37.

22. Ruchholtz S, Lefering R, Paffrath T, et al. Reduction in mortality of severely injured patients in Germany. Dtsch Arztebl Int 2008; 105: 225-231.

23. Rutherford EJ, Livengood J, Higginbotham M, et al. Efficacy and safety of pneumococcal revaccination after splenectomy for trauma. J Trauma 1995; 39: 448-452.

24. Schurr MJ, Fabian TC, Gavant M, et al. Management of blunt splenic trauma: computed tomographic contrast blush predicts failure of nonoperative management. J Trauma 1995; 39: 507-513.

25. Shapiro MJ, Krausz C, Durham RM, et al. Overuse of splenic scoring and computed tomographic scans. J Trauma 1999; 47: 651-658.

26. Shatz DV, Schinsky MF, Pais LB, et al. Immune response of splenectomized trauma patients to the 23 -valent pneumococcal polysaccharide vaccine at 1 versus 7 versus 14 days after splenectomy. J Trauma 1998; 44: 760-766. 
27. Sperling P, Hartmann J. Aktueller Stand der Fibrinklebung in der Therapie der Milzverletzung. Chir Gastroenterol 2001; 17: 28-32.

28. Stein DM, Scalea TM. Nonoperative management of spleen and liver injuries. J Intensive Care Med 2006; 21: 296-304.

29. Vincent JL, Moreno R, Takala J, et al. The SOFA (Sepsisrelated Organ Failure Assessment) score to describe organ dysfunction/failure. On behalf of the Working Group on Sepsis-Related Problems of the European Society of Intensive Care Medicine. Intensive Care Med 1996; 22: 707-710.

30. Wiseman J, Brown CV, Weng J, et al. Splenectomy for trauma increases the rate of early postoperative infections. Am Surg 2006; 72: 947-950.
31. Woltmann A, Trentz O. Abdominalverletzungen. Trauma Berufskrankh 2004; 6: 73-83.

Received: November 2, 2009 / Accepted: November 13, 2009

Address for correspondence:

Sven Lendemans, M.D.

Department of Trauma Surgery

University Hospital Essen

Hufelandstrasse 55, 45122 Essen, Germany

Phone: +49 (201) 72384105

Fax: +49(201) 7231397

E-mail: sven.lendemans@uk-essen.de 